\title{
Modified Brown-Séquard syndrome following coronary artery bypass graft: case report
}

\author{
M H Gottesman MD,${ }^{1}$ Ibrahim Saraya MD,${ }^{2}$ Franesco Tenti MD $^{3}$ \\ The Divisions of ${ }^{1}$ Neurology, ${ }^{2}$ Cardiology, ${ }^{3}$ Thoracic-Cardiovascular Surgery, Winthrop \\ University Hospital, Clinical Campus, School of Medicine, State University of New York \\ at Stony Brook, 259 First Street, Mineola, NY 11501, USA.
}

\begin{abstract}
A 75 year old man underwent a triple coronary artery bypass graft performed with intra-aortic balloon pump assistance. Left leg weakness developed on the first postoperative day, progressive worsening occurred over the next $2 \frac{1}{2}$ weeks, finally culminating in a modified Brown-Séquard pattern of deficit. No etiology was found. Previous reports of spinal cord injury following coronary artery bypass graft are reviewed and the microcirculation of the spinal cord is discussed.
\end{abstract}

Key words: spinal cord infarct; Brown-Séquard syndrome; coronary artery bypass surgery; intra-aortic balloon pump.

\section{Introduction}

Coronary artery bypass surgery (CABG), when performed with or without the aid of an intra-aortic balloon pump (IABP), is rarely complicated by spinal cord dysfunction. We found only 8 reported cases, ${ }^{1-7}$ in 6 of which the IABP was used. The onset of the deficit ranged from 15 minutes after insertion of the $\mathrm{IABP}^{1}$ to the seventh postoperative day. ${ }^{4}$ We report a case of modified Brown-Séquard syndrome complicating a CABG performed with IABP assistance.

\section{Case report}

A 75 year old man experienced sudden chest pain and was admitted to another hospital. His past medical history included a myocardial infarction 2 years ago, hypertension, gout and mild renal insufficiency. He had recently stopped smoking. There were no past symptoms referable to the nervous system. Other than his cardiac status, the general physical examination was normal. He was mentally clear and the remainder of the neurological examination was reported as 'normal'.

Coronary angiography was performed on the day of admission and revealed extensive triple vessel disease, including severe nar- rowing of the left main coronary artery. Despite intravenous nitrogylcerin the patient continued to have chest pain and became hypotensive. ST depression was present in multiple EKG leads. Two days after admission an IABP (manufactured by the Datascope Corporation, Paramus, New Jersey, USA), was inserted under fluoroscopy without any untoward effects, and anticoagulation was initiated. The top of the balloon was beneath the left subclavian artery, the bottom of the balloon was above the renal arteries.

On the third day the patient was transferred to Winthrop University Hospital and a triple coronary artery bypass was performed. No neurological deficits were noted prior to the surgery. No postoperative hypotension occurred. Sixteen hours postoperatively the IABP was removed. The patient was alert and oriented, but by the end of the first postoperative day his ability to elevate the left lower limb was impaired; no sensory abnormalities were present. All peripheral pulses were intact and there was no clinical or laboratory evidence of embolization.

On the third postoperative day the left leg weakness persisted and a left Babinski response was noted; the right leg was normal. All deep tendon reflexes were present and symmetric. Fecal and urinary 
incontinence and diminished anal sphincter tone were noted on the eighth postoperative day. Bilateral Babinski responses and mild right leg weakness were present on postoperative day 11 . Temperature and pain sensation were diminished in the right leg.

Two and a half weeks postoperatively the patient's neurological status stabilized and the following were present: (1) marked weakness of the left lower limb; (2) hypesthesia to pinprick and diminished temperature perception from the waist down on the right; (3) position sense errors of the left toes; (4) vibratory sense impairment from the waist down on the left; (5) mild weakness of the right lower limb; (6) bilateral Babinski responses; and (7) urinary and fecal incontinence. These findings are highly suggestive of a predominately unilateral injury to the spinal cord resulting in a pattern of deficit similar to the BrownSéquard syndrome. Neurological investigation during this time included 2 normal CTs of the brain, normal CT of the chest and abdomen with particular reference to the thoracic and abdominal aorta, and a normal MRI of the spine and spinal cord. The patient made a slow gradual improvement. Currently, ( 15 months after the surgery) he is able to walk with a cane and has partial control of his sphincters.

\section{Discussion}

None of the 8 previously reported cases of spinal cord dysfunction associated with CABG resulted in a Brown-Séquard pattern of deficit. All patients developed profound bilateral leg weakness. Only 3 cases included an examination of posterior column function; in one case it was intact ${ }^{5}$ and in 2 it was abnormal. ${ }^{6}$ We postulate a disturbance of a spinal artery in the thoracic region affecting the circulation of the left side of the spinal cord. The entire left half of the spinal cord, not just the anterior portion supplied by the anterior spinal artery, must have been involved to account for the posterior column dysfunction present in this case. Presumably one of the left sided spinal arteries, which in turn divides into anterior and posterior radicular branches, ${ }^{8}$ was compromised, producing most of the damage in the left side of the cord. Interestingly, the majority of spinal arteries supplying the thoracic and lumbar spinal cord, as well as the artery of Adamkiewicz, a large anterior radicular artery, are located on the left side of the cord. The left sided radicular arteries also supply the left lateral aspect of the cord via their anastomotic surface vessels, the arterial vasocorona. ${ }^{8}$ Involvement of these vessels, which supply half of the spinal cord, could result in a Brown-Séquard syndrome.

Although the actual pathological basis of this patient's spinal cord dysfunction was not determined, there are several possible explanations. Aortic dissection or embolization of an atherosclerotic plaque, perhaps compounded by unrecognized hypotension or movement of the IABP, may have occurred. Blood vessel spasm, local inflammatory response, or repetitive episodes of embolization may have contributed to the progressive nature of the deficit. In addition, this patient may have an anomalous spinal cord vasculature and be especially vulnerable to ischemia.

We believe that this is the first report of progressive neurological dysfunction resulting in a modified Brown-Séquard syndrome following $\mathrm{CABG}$ surgery. The specific role played by the IABP in producing this neurological complication is unclear. It does not appear possible prospectively to identify patients who are likely to have this serious complication. Free radical scavenging agents and calcium channel blockers are being evaluated as therapeutic agents for cerebral infarction. The former inhibit the inflammatory response and the latter diminish vasospasm. Perhaps these agents will have a therapeutic role in spinal cord ischemia and diminish the severity of this rare but devastating complication of coronary artery bypass surgery.

\section{References}

1 Rose DM, Jacobowitz IJ, Acinapura AJ, Cunningham JN (1984) Paraplegia following percutaneous insertion of an intra-aortic balloon. J Thorac Cardiovasc Surg 87: 788-789. 
2 Harris RE, Reimer KA, Crain BJ, Becsey DD, Oldham HN (1986) Spinal cord infarct following intra-aortic balloon support. Ann Thorac Surg 42: 206-207.

3 Seifert PE (1986) Late paraplegia resulting from intra-aortic balloon pump. Ann Thorac Surg 41: 700.

4 Scott IR, Goiti JJ (1985) Late paraplegia as a consequence of intra-aortic balloon pump support. Ann Thorac Surg 40(3): 300-301.

5 Archer AG, Choyke PL, Zeman RK, Green CE, Zuckerman M (1986) Aortic dissection following coronary artery bypass surgery: diagnosis by CT. Cardiovasc Intervent Radiol 9: 142-145.

6 Kewalramani LS, Orth MS, Katta SR (1981) Atraumatic ischaemic myelopathy. Paraplegia 19: 352-362.

7 Tyras DH, Willman VL (1987) Paraplegia following intra-aortic balloon assistance. Ann Thorac Surg 25(2): 164-166.

8 Carpenter MB, Sutin J (1983) Human Neuroanatomy. Williams and Wilkins, Baltimore: 708-711. 